Philosophie ANTIQUE
Philosophie antique

Problèmes, Renaissances, Usages

$13 \mid 2013$

Analogie et connaissance

\title{
Anaxagore : analogie, proportion, identité
}

\section{Claire Louguet}

\section{(2) OpenEdition}

\section{Journals}

Édition électronique

URL : https://journals.openedition.org/philosant/903

DOI : 10.4000/philosant.903

ISSN : 2648-2789

\section{Éditeur}

Éditions Vrin

\section{Édition imprimée}

Date de publication : 1 novembre 2013

Pagination : 117-145

ISBN : 978-2-7574-0591-8

ISSN : 1634-4561

\section{Référence électronique}

Claire Louguet, "Anaxagore : analogie, proportion, identité », Philosophie antique [En ligne], 13 | 2013, mis en ligne le 01 novembre 2018, consulté le 02 décembre 2022. URL : http://

journals.openedition.org/philosant/903; DOI : https://doi.org/10.4000/philosant.903

\section{(c) $(1)$}

Creative Commons - Attribution - Pas d'Utilisation Commerciale - Pas de Modification 4.0 International - CC BY-NC-ND 4.0

https://creativecommons.org/licenses/by-nc-nd/4.0/ 


\section{ANAXAGORE : ANALOGIE, PROPORTION, IDENTITÉ Claire LOUGUET \\ UMR Savoirs, Textes, Langage/Université Charles-de-Gaulle Lille 3}

RÉSUMÉ. Alors que l'analogie (qu'elle soit entendue au sens propre comme égalité de rapports ou au sens faible comme métaphore ou comparaison) est largement répandue dans la philosophie ancienne (chose évidente quand on lit les autres présocratiques, Platon et Aristote), Anaxagore ne fait usage d'aucun type d'analogie. Il met en revanche l'accent sur un autre type de relation que nous appellerons l'« homologie », terme par lequel nous entendons une relation d'identité entre deux choses. De plus, alors que l'analogie était habituellement utilisée dans un but heuristique, nous verrons que l'homologie est à chaque fois révélée au moyen d'une inférence. Le but de cet article est d'essayer de montrer que ces deux faits sont liés et qu'Anaxagore a délibérément choisi de remplacer l'analogie par l'homologie afin de rendre manifeste, grâce à l'écriture, une loi universelle sous-jacente aux phénomènes sensibles, à savoir l'irréductibilité du mélange universel, qui s'avère être un obstacle à l'activité de l'Intellect cosmique autant que sa condition de possibilité.

SUMMARY. Whereas analogy (be it understood either in the proper sense as an equality between two ratios or in a wider sense as metaphor or comparison) is widespread in ancient philosophy (which is self-evident when we read the other Presocratics, Plato and Aristotle), Anaxagoras does not use any analogy whatsoever. Instead, he focuses on another kind of relationship we will call "homology", by which we mean a relation of identity between two things. Moreover, whereas analogy would be used for heuristic purpose, we will see that homology is in each case revealed through inference. The aim of this paper is to try to show that these two facts are interconnected and that Anaxagoras has intentionally chosen to replace analogy with homology in order to make clear, through literary device, a universal law which underlies the phenomena, namely the irreducibility of the universal mixture, which turns out to binder the activity of the cosmic Intellect as much as it enables it. 



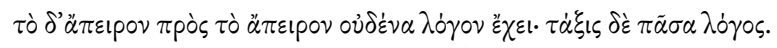
ARISTOTE, Physique, VIII, 1, 252a13 sq.

Je dédie cet article à Diego Lanza'.

Qu'on la prenne dans son acception stricte (égalité de rapports) ou dans son sens dérivé (métaphore, comparaison), l'analogie révèle un ordre entre deux domaines distincts qu'elle met en rapport. Qu'elle soit instrument de connaissance ou moyen d'exposer une idée qui serait sans cela trop abstraite, elle constitue un principe d'intelligibilité : la mise en rapport permet de ramener l'autre au même et révèle des structures communes. Socrate, Platon et Aristote y ont eu abondamment recours et les présocratiques également. Dans ce contexte, Anaxagore fait figure d'exception. Il semble en effet n'y avoir chez lui aucune analogie (ni au sens strict ni au sens faible). En revanche, son système est traversé par la figure de l'homologie.

Par «homologie », nous entendrons ici une similitude forte (pour ne pas dire une relation d'identité) entre deux ordres, deux états du monde ou de la matière. Entendue en ce sens, l'homologie est l'expression de la répétition du même au premier degré. On pourrait dire que, alors que l'analogie exprime une égalité de second ordre (une égalité de rapports), l'homologie exprime une égalité ou une identité de premier ordre.

Le but de cet article est de tenter de comprendre pourquoi Anaxagore a préféré l'homologie à l'analogie. L'hypothèse qui sera proposée ici est que cette absence n'est pas fortuite mais qu'elle résulte au contraire d'un choix

1. Les réflexions exposées dans cet article ont pour une large part été suscitées par deux observations très judicieuses faites par Lanza 1965, entre lesquelles il n'établissait pas de lien : l'absence de métaphore chez Anaxagore et l'originalité de son dualisme (l'altérité de l'Intellect tenant au fait qu'il est de façon absolue ce que les autres choses ne sont jamais que de façon relative). Pour une discussion des thèses originales de Lanza, voir Louguet 2013, dont la présente étude constitue un prolongement. 
pour ainsi dire stylistique lié à sa conception du monde, ou, pour le dire autrement, que son écriture est une illustration performative de la façon dont il conçoit les choses.

\section{L'analogie introuvable}

\subsection{L'analogie au sens strict}

Par analogie au sens strict, nous entendons ce que Platon et Aristote désignent par le terme à $\nu \alpha \lambda \circ \gamma i \alpha$, à savoir une égalité (à l'origine géométrique) de rapports, du type $\mathrm{A} / \mathrm{B}=\mathrm{C} / \mathrm{D}$ ( $\mathrm{A}$ est à $\mathrm{B}$ ce que $\mathrm{C}$ est à $\mathrm{D}$ ). Dans tout le corpus anaxagoréen (fragments et témoignages), on ne relève qu'une seule analogie de ce type :

Anaxagore dit dans son Sur la Nature que ce qu'on appelle "lait d'oiseau" est le blanc qui est dans les œufs. (Athénée, Deipn. Epit. II, 57b = 59B22 $\mathrm{DK}^{2}$.)

Que l'on attribue ou non $\tau \grave{o} \kappa \alpha \lambda \circ \dot{\mu \varepsilon v \nu o v ~ a ̀ ~ A n a x a g o r e ~ n ' a ~ p a s ~ d ' i n c i d e n c e ~}$ sur notre propos ${ }^{3}$. Ce qui importe en revanche, c'est que nous avons là l'analyse d'une expression métaphorique qui repose sur une analogie au sens fort (implicite) : le blanc d'œuf est aux oiseaux ce que le lait est aux mammifères, à savoir l'élément nutritif du petit. La section du texte source dans laquelle ce fragment est cité est consacrée aux œufs, mais rien ne nous permet de déterminer quels en étaient le contexte et la finalité dans le traité d'Anaxagore. Du reste, il n'est pas absolument certain qu'il en soit bien l'auteur, car, comme l'a justement remarqué Sider ${ }^{4}$, c'est à Alcméon qu'Aristote attribue la thèse selon laquelle l'élément nutritif de l'œuf est le blanc (Génération des animaux, III, 2, 752b25).

Quoi qu'il en soit, comme nous l'avons dit, ce fragment constitue le seul cas d'analogie au sens fort dans tout le corpus anaxagoréen, et étant donné que le contexte ne nous donne aucun élément, son interprétation ne va pas de soi.

\subsection{L'analogie au sens faible (métaphore et comparaison)}

Bien qu'Aristote rapporte la métaphore à l'analogie au sens strict, c'està-dire reposant sur une relation à quatre termes (le «bouclier de Dionysos » peut désigner sa coupe, Poétique, 1457b, le bouclier étant à Arès ce

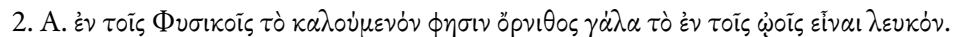

3. Pour Brennan 1995, cela veut dire « l'expression », le « proverbe », mais cela pourrait simplement vouloir dire « pour ainsi dire ». Remarquons, cela dit, qu'Aristote (dans le texte cité infra) emploie lui-même l'expression $\tau$ ò $\kappa \alpha \lambda \circ u j \mu \varepsilon v o ́ v ~ \gamma \dot{\alpha} \lambda \alpha$ pour désigner le lait des mammifères, ce qui est assez étrange.

4. Sider 2005, p. 169-170. 
que la coupe est à Dionysos), alors que la comparaison établit un rapport entre deux termes (que nous qualifions couramment d'analogique par abus de langage), nous les traiterons ensemble dans ce qui suit comme des «analogies au sens faible» ou images, Anaxagore ne recourant au demeurant ni aux unes ni aux autres.

Maints fragments et témoignages attestent l'importance de l'image chez les penseurs présocratiques. Dans ce contexte, le style d'Anaxagore apparaît très différent. Il ne fait en effet aucun usage de comparaisons ou de métaphores. À une exception près :

Les choses qui sont dans le monde un ne sont pas séparées les unes des autres ni coupées à la hache, ni le chaud du froid, ni le froid du chaud. (B8, cité par Simplicius, in Phys., 175, 11 et 176, 28.)

Cependant, notons qu'Anaxagore fait ici un usage négatif de la métaphore, comme s'il s'agissait là d'un refus performatif de l'analogie.

Le seul point sujet à caution pourrait être le terme $\sigma \pi \dot{\varepsilon} p \mu \alpha$. En effet, les interprètes ont souvent soutenu que ce mot, qui n'apparaît que dans le fragment B4, était un terme technique par lequel Anaxagore désignait les « éléments » invisibles en raison de leur petitesse. Il aurait utilisé de façon métaphorique un terme à sa disposition pour désigner une chose sans nom (qu'Aristote désigne au moyen du terme « homéomère »). Cependant, se fondant sur une étude philologique du terme $\sigma \pi \dot{p} p \mu \alpha$ à l'époque archaïque, Lanza a montré que son usage n'était jamais métaphorique, et, en résumant ses résultats, il commence par une remarque fort intéressante concernant l'absence de métaphore chez Anaxagore : « non seulement la métaphore en général est étrangère au style anaxagoréen, et elle le serait d'autant plus pour une innovation d'une telle importance, mais dans ce cas précis la métaphore aurait même été incompréhensible, parce que le grec ne connaît pas d'usage métaphorique de $\sigma \pi \dot{\varepsilon} p \mu \alpha$ comparable à l'usage latin de semen $^{6}$. » Quoi qu'il en soit, et bien au-delà du problème qui nous occupe, le caractère métaphorique de l'usage technique des termes serait encore à prouver (il est peu vraisemblable qu'Aristote pense au bois à chaque fois qu'il emploie vं $\lambda \eta \eta$.

Comment donc expliquer cette absence d'image ? On pourrait soutenir que le choix d'un style sec constitue un geste philosophique fort et témoigne d'une volonté d'exprimer les idées d'une façon plus rationnelle et abstraite que ne le permet le registre poétique. Cependant, cette hypothèse n'est pas entièrement satisfaisante. Platon et Aristote, qui sont incontestablement plus abstraits que n'importe quel présocratique, n'ont pas fait 
l'économie de l'image et de l'analogie ; bien au contraire, ces procédés sont de précieux adjuvants à la clarté de ce qu'ils exposent et, bien souvent, ils donnent à l'interlocuteur ou au lecteur des repères familiers qui lui permettent de progresser vers l'inconnu. Rien de tel chez Anaxagore?

Nous verrons dans ce qui suit que, si l'analogie est absente, l'homologie, elle, est omniprésente. Nous constaterons que, contrairement à l'analogie telle qu'elle peut être employée chez Platon et Aristote, l'homologie n'a pas une fonction heuristique, mais qu'elle est au contraire à chaque fois le demonstrandum du raisonnement. Nous essayerons par la suite d'expliquer pourquoi ce choix de l'homologie a rendu impossible le recours à l'analogie.

\section{Homologies}

Par « homologie », nous entendrons une comparaison entre deux états du monde ou de la matière, mais une comparaison qui doit être prise au pied de la lettre (c'est-à-dire qui n'a aucune portée métaphorique) et qui révèle une loi universelle. Ainsi, Anaxagore dit que maintenant comme à l'origine tout est mélangé (B6) et inversement (témoignages); que ailleurs comme ici la séparation se produit (B4a); et que par rapport à elle-même chaque chose est grande et petite (B3). Un trait commun relie toutes ces homologies : toutes sont descriptives et sont le résultat d'un raisonnement (aucune ne constitue un procédé heuristique), ce qui pourrait être une façon de comprendre le fragment B21a (ö $\psi \varsigma \tau \tau \tilde{\omega} \nu \dot{\alpha} \delta \dot{\eta} \lambda \omega \nu \tau \dot{\alpha} \phi \alpha \iota v \dot{\mu} \mu \varepsilon \nu \alpha$ : « les phénomènes sont la vision de choses non manifestes »). En effet, l'homologie n'est pas manifeste; elle ne se révèle que par le biais du raisonnement.

\subsection{L'homologie quantitative}

Le fragment B3 établit une égalité quantitative du grand et du petit qui traduit une égalité de structure. On pourrait parler d'isomorphisme quantitatif, ou de modèle fractal, mais à condition de ne pas entendre par là la réplication d'une forme. Il s'agit plutôt d'une propriété de ce qu'Aristote appellera plus tard le continu, raison pour laquelle nous préférerons parler d'homologie quantitative :

Du petit, en effet, il n'y a pas de plus petit absolu, mais il y a toujours un plus petit encore (car il est impossible que ce qui est ne soit pas) - mais du grand aussi il y a toujours un plus grand encore. Et il est égal en quantité au

7. Sedley 2007 a proposé une belle lecture métaphorique : l'Intellect aurait les caractéristiques d'un fermier qui pourvoit aux besoins de ses bêtes et de ses plantes. Cette interprétation judicieuse donne à voir, et il y a quelque chose de rassurant à ramener l'activité de l'Intellect à une activité familière. Le problème est qu'Anaxagore lui-même ne fait pas explicitement cette analogie. 
petit, et, relativement à elle-même, chaque chose est à la fois grande et petite. (B3, cité par Simplicius in Phys. 164, 16 ${ }^{8}$.)

La prémisse est énoncée dans la parenthèse : «il est impossible que ce qui est ne soit pas ». De cette prémisse d'inspiration parménidienne, Anaxagore conclut qu'il n'y a pas de plus petit absolu, c'est-à-dire pas de minimum quantitatif en deçà duquel il n'y aurait rien. L'absence de plus grand absolu n'est pas argumentée ; sans doute la thèse de l'inexistence d'un minimum quantitatif est-elle plus importante (de fait, c'est elle qui sera convoquée dans le fragment B6).

L'égalité du grand et du petit ne peut pas être une égalité en grandeur des choses telles que nous les percevons. Anaxagore dit en B1 que l'air et l'éther sont « les plus grandes choses en quantité et en grandeur »; il dit par ailleurs que le soleil est plus grand que le Péloponnèse ${ }^{9}$ et en B12 que l'Intellect commande toutes les choses qui ont une âme, « aussi bien les grandes que les petites $\gg$. Il ne met donc pas en cause le fait qu'il existe des choses plus ou moins grandes. Il parle en revanche d'une égalité du grand et du petit en quantité $(\pi \lambda \tilde{\eta} \theta \circ \varsigma)$ : comme le dira le fragment B6, « les parts du grand et celles du petit sont égales en quantité ». Il s'agit vraisemblablement d'une égalité en nombre de parties ou de divisions. Comme l'a suggéré $\operatorname{Sider}^{10}$, il y a autant de lignes qui peuvent couper un petit segment qu'un grand segment, à savoir une infinité. De même, on pourrait dire que le soleil, qui est plus grand que le Péloponnèse, pourrait être divisé en au moins autant de parts que lui, et que ce nombre ne varie pas du fait qu'on le voie plus petit.

Ce qui est décrit dans ce fragment est un système où l'échelle indéfinie, et même infinie, des grandeurs comparées invalide toute comparaison quantitative, tout rapport de grandeur. Le relativisme absolu aboutit à l'annihilation du relatif. Aussi le grand «est égal en quantité au petit». On aurait pu s'attendre à une conclusion du type « chaque chose est à la fois plus grande que ce qui est plus petit qu'elle et plus petite que ce qui est plus grand $\gg$. Mais la conclusion qu'énonce Anaxagore est bien différente, et plus forte : c'est par rapport à elle-même ( $\pi$ pòs $\dot{\varepsilon} \alpha \nu \tau \dot{0})$ que « chaque chose est à la fois grande et petite ». L'autre est intégré dans le même. Il n'est pas à chercher à l'extérieur. L'analogie est auto-référentielle. L'égalité de rapports (+ grand/grand $=$ petit/ + petit $)$ aboutit à une égalité du grand et du petit, qui elle-même débouche sur la conclusion paradoxale que $\mathrm{A} / \mathrm{A}>1$ et

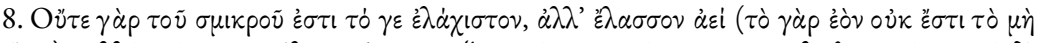

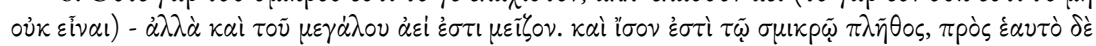

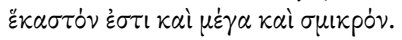

9. A 42 et A72 DK.

10. Sider 2005 ad loc. 
$\mathrm{A} / \mathrm{A}<1$ à la fois, c'est-à-dire à l'évanescence de l'identité, ou du moins de la quantité assignable ${ }^{11}$. «Plus grand », «plus petit» et « égal » peuvent relier toutes quantités. La grande absente de ce fragment est l'égalité à soi, comme si Anaxagore avait anticipé la thèse d'Aristote selon laquelle est infini ce dont une partie est toujours en dehors, thèse qui ruine la possibilité d'une totalité infinie. Le caractère paradoxal et contrefactuel des conclusions de B3 est peut-être une façon d'exprimer ces choses qui ne pouvaient être exprimées autrement.

\subsection{L'homologie spatiale}

Dans le fragment B4a, Anaxagore soutient que la séparation a également (eu) lieu ailleurs que chez nous.

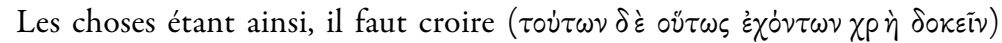
que, dans toutes les choses qui s'agrègent, se trouvent de nombreuses choses de toutes sortes et des semences de toutes choses qui ont des formes, des couleurs et des saveurs de toutes sortes; que des hommes se sont solidifiés, ainsi que tous les autres êtres vivants qui ont une âme ; et que ces hommes ont des villes qu'ils habitent ensemble, et des œuvres fabriquées, comme chez nous ( $\check{\sigma \tau \tau \varepsilon \rho} \pi \alpha \rho ' \dot{\eta} \mu \tilde{\nu})$, et qu'ils ont un soleil, une lune et les autres <astres>, comme chez nous ( $\check{\sigma \pi \varepsilon \rho} \pi \alpha \rho^{\prime} \dot{\eta} \mu \tilde{\imath} \nu$ ), et que la terre fait croître pour eux de nombreux fruits de toutes sortes dont ils récoltent les plus utiles et les emploient chez eux. Voici donc ce que je dis à propos de la séparation : que cette séparation pourrait avoir lieu (ou aurait lieu) non seu-

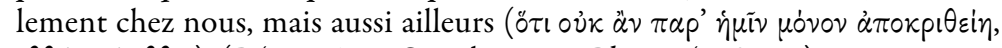

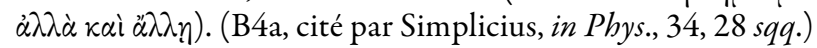

Sans entrer dans les débats interprétatifs que ce fragment a suscités ${ }^{12}$, nous nous contenterons de relever deux points importants pour le propos qui nous occupe : l'anaphore de $\check{\omega} \sigma \pi \varepsilon p \pi \alpha \rho^{\prime} \dot{\eta} \mu \tilde{\tau}$, qui insiste sur l'homologie entre ailleurs et chez nous, et le fait que la thèse de l'homologie soit le résultat d'une inférence.

Quel que soit l'ailleurs dont parle Anaxagore, il est remarquable que nature et culture y soient « comme chez nous ». En quelques lignes, sont présentés des points clés d'un traité $\pi \varepsilon p i ~ \phi \dot{\nu} \sigma \varepsilon \omega s:$ on y retrouve, bien que l'ordre canonique des doxographes ne soit pas respecté, les principes élémentaires, la cosmologie, la zoogonie et l'anthropogonie, la croissance des plantes, et la culture (politique, économie domestique, agriculture, artisanat). Toutes choses qui s'observent ici, mais qui se passent aussi ailleurs

11. Pepe 2002 a montré, en une étude très convaincante, ce que le Philèbe devait au fragment B3.

12. Les différentes interprétations anciennes et modernes sont présentées dans Louguet 2002. Sedley 2007 a proposé une nouvelle lecture. 
« comme chez nous », y compris la séparation (processus cosmogonique), qui aura eu lieu «non seulement chez nous, mais aussi ailleurs ». Ainsi, loin qu'il soit question d'analogie, cet ailleurs en miroir est homologue, pour ne pas dire identique, à notre monde. Qu'il soit réplique ou mise en abyme du nôtre, ce monde-là est le même, malgré l'adverbe qui sert à le désigner $(\ddot{\alpha} \lambda \lambda \eta \eta)$.

Cette homologie est inférée, comme l'indique le début du fragment :

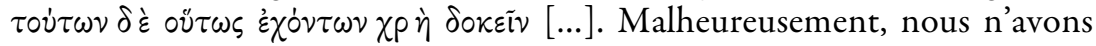
aucun élément permettant de savoir à quoi renvoie la prémisse. Simplicius, avant de citer le fragment, indique qu'il était situé non loin du début du

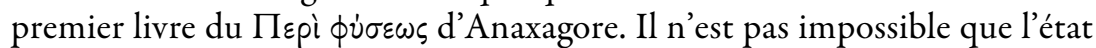
de choses auquel la prémisse fait référence soit le mélange universel de B1. Par conséquent, où que la séparation-agrégation ait lieu, les mêmes choses se produiront, les mêmes causes (mélange universel et processus d'association-dissociation) engendrant les mêmes effets. Autrement dit, tout agrégat contient le même programme et porte en germe tout ce que nous connaissons, à l'identique.

\subsection{L'homologie temporelle}

\subsubsection{La situation présente est homologue à la situation originelle}

Maintenant comme à l'origine, soutient Anaxagore en B6, les choses sont mélangées ( $c f$. aussi B12, in fine). Cette thèse est la conclusion d'un raisonnement prenant comme prémisse un point acquis en $\mathrm{B} 3$.

Puisque les parts du grand et celles du petit sont égales en quantité, ainsi aussi toutes les choses pourront être dans toute chose. Et il n'est pas possible non plus < pour les choses> d'exister séparément, mais toutes choses ont leur part dans toute chose. Puisque le plus petit ne peut être, il ne peut ni être séparé ni être par lui-même, mais, tout comme à l'origine, maintenant encore, toutes choses sont ensemble. Et dans toutes choses se trouvent de nombreuses choses, et des choses qui se séparent en quantité égale dans les plus grandes et dans les plus petites. (B6, cité par Simplicius, in Phys. 164, 25 sqq..$^{13}$ )

Le raisonnement s'appuie sur deux thèses de B3 : l'égalité en quantité du grand et du petit, et l'impossibilité d'un minimum absolu. Ces deux thèses, bien qu'elles soient toutes les deux précédées d'une conjonction causale, ne sont pas utilisées de la même façon.

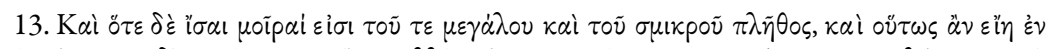

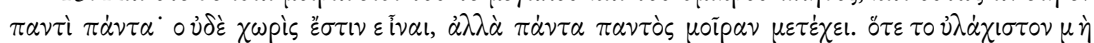

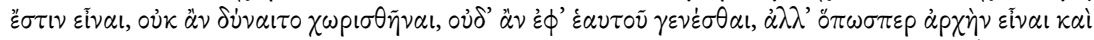

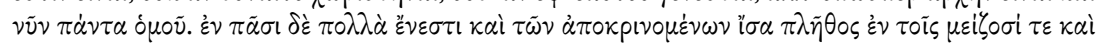
$\dot{\varepsilon} \lambda \dot{\alpha} \sigma \sigma \circ \sigma$. 
La première thèse («il [scil. le grand] est égal en quantité au petit », B3) est reprise ici pour exprimer la condition de possibilité du mélange universel. Elle est formulée en des termes qui sont néanmoins moins ambigus qu'en B3: «les parts du grand et celles du petit sont égales en quantité $\gg$. Ainsi, le mélange sera possible à quelque échelle que ce soit : la divisibilité n'est pas l'apanage du grand; le petit est tout autant divisible que lui. Pour le moment, il n'est question que de quantité (comme en B3), et il semble y avoir un saut entre la condition de possibilité (l'égalité quantitative des parts du grand et du petit) et ce qu'elle rend possible (le mélange universel).

La deuxième thèse (« du plus petit (...) il n'y a pas de plus petit absolu, mais toujours un plus petit encore », B3) est rappelée ici sous une forme ramassée («le plus petit ne peut être »). Elle est utilisée ici comme prémisse du raisonnement établissant l'impossibilité de l'existence séparée du plus petit. Autrement dit, alors que la première section rendait le mélange possible, celle-ci élimine la possibilité qu'il ne soit pas, et le rend donc nécessaire. L'impossibilité de l'existence séparée d'un minimum rend impossible un état pur. Elle fait ainsi obstacle à l'activité discriminante de l'Intellect et abolit en quelque sorte le temps, choses que B12 illustre sans ambiguïté :

Alors que toutes les autres choses ont une part de toute chose, l'Intellect est infini, souverain absolu, et il n'est mêlé d'aucune chose, mais il est le seul à être lui-même par lui-même. En effet, s'il n'était pas par lui-même, mais était mêlé de quelque chose d'autre, il aurait une part de toutes choses, s'il était mêlé d'une. En effet, dans toute chose il y a une part de toute chose, comme je l'ai dit précédemment [B11], et les choses mêlées à lui lui feraient obstacle, de sorte qu'il ne pourrait commander aucune chose comme il le fait en étant seul par lui-même. En effet, il est la plus subtile et la plus pure de toutes choses, et il a une connaissance absolue de toute chose, et a la plus grande force ; et toutes les choses qui ont une âme, les plus grandes comme les plus petites, l'Intellect les commande. Et l'Intellect a commandé l'ensemble de la révolution, de sorte qu'il y eût révolution au commencement. Et d'abord, la révolution a commencé par le petit, et puis la révolution augmente, et elle augmentera encore. Et les choses qui se mélangent, ainsi que celles qui se séparent et se distinguent, l'Intellect les a toutes connues. Et toutes les choses qui sont maintenant, comment elles devaient être, comment elles étaient et comment elles seront, l'Intellect les a toutes mises en ordre, ainsi que cette révolution qu'effectuent maintenant les astres, le soleil, la lune, et l'air et l'éther qui se séparent. Et cette révolution a produit la séparation. Et du rare se sépare le dense, du froid le chaud, de l'obscur le lumineux et de l'humide le sec. Mais il y a beaucoup de parts de beaucoup de choses. Mais rien ne se sépare ni ne se distingue complètement d'autre chose sauf l'Intellect. Et l'Intellect est tout entier sem- 


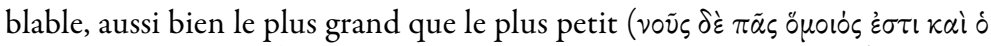

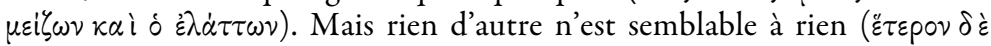

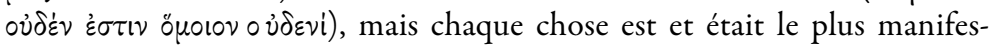

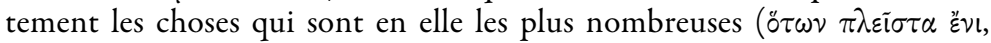

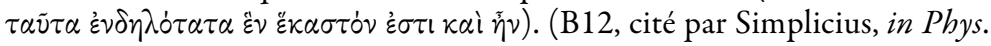
164,$24 ; 156,13$.)

L'Intellect initie et poursuit un processus de séparation, et on pourrait aller jusqu'à dire qu'il crée le temps en même temps que le mouvement (Aristote dit, lui, en Physique VIII, 1, que le mélange est demeuré immobile pendant «un temps infini » avant l'intervention de l'Intellect) : il introduit la temporalité avec le processus qu'il inaugure, comme le montre l'usage des temps en B12 (passé, présent et futur). Cependant, les dernières lignes insistent sur le fait que rien ne se sépare (et donc n'existe à l'état séparé) hormis l'Intellect - l'homogénéité des choses, le cas échéant, n'étant que d'ordre phénoménal et ne s'expliquant que par la prédominance. Ce conflit, pour ainsi dire, entre mouvement et inertie, entre processus et immobilité, entre temporalité et atemporalité, est traduit par le style même de B12: le récit linéaire de la séparation opérée par l'Intellect est enchâssé dans une composition circulaire qui borne la progression de la séparation, ou même la contredit. Mais, loin que cette composition circulaire signifie la clôture du système sur lui-même en un tout achevé, elle exprime en fait la condition de possibilité de la continuation indéfinie du processus de séparation. Nous allons y revenir.

\subsubsection{La situation originelle est homologue à la situation présente}

L'homologie temporelle entre maintenant et l'origine (mélange universel) est établie explicitement par Anaxagore, comme nous venons de le voir. Mais comment en est-il venu à l'hypothèse du mélange originel ? Aristote conjecture qu'il l'a inférée à partir de l'observation des phénomènes. Dans l'argument tel qu'il le reconstruit, c'est parce qu'il voyait que n'importe quoi naît de n'importe quoi qu'Anaxagore a concu qu'il y eut un temps où toutes choses étaient ensemble, comme elles le sont maintenant encore dans toute chose donnant naissance à une chose qui est autre qu'elle.

La lecture conjointe de deux textes de la Physique nous permettra de comprendre comment Aristote reconstruisait l'argument.

Dans le premier texte, la conclusion du raisonnement est que « tout est mêlé à tout » :

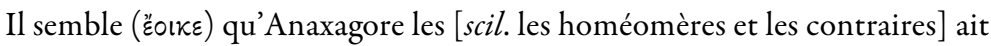
ainsi crus infinis (1) parce qu'il estimait vraie l'opinion commune des physiciens selon laquelle rien ne naît de ce qui n'est pas (c'est en effet pour cela qu'ils disent ceci : toutes choses étaient ensemble, et la génération de telle chose est en réalité une altération, ou, selon d'autres, une association et 
une dissociation), et aussi (2) du fait que les contraires naissent les uns des autres : c'est donc qu'ils étaient déjà présents. En effet, s'il est nécessaire que tout ce qui naît naisse soit de choses qui sont soit de choses qui ne sont pas, et (1) s'il est impossible que ce soit de choses qui ne sont pas (c'est de fait une opinion qui fait l'unanimité chez tous les physiciens), ils [scil. les anaxagoréens] ont pensé que, dès lors, c'est l'autre <hypothèse envisagée> qui s'ensuit nécessairement, à savoir que la naissance a lieu à partir de choses qui sont et qui sont déjà présentes, mais qui nous sont imperceptibles ( $\dot{\alpha} \alpha \omega \sigma \theta \dot{\eta} \tau \omega \nu \dot{\eta} \mu \tilde{\imath} \nu)$ en raison de la petitesse de leur masse. C'est pourquoi ils disent que (3) tout est mêlé à tout, parce qu'ils voyaient $(\dot{\varepsilon} \omega \omega \omega \nu)$ que (2') tout naît de tout ; mais, <toujours selon eux>, les choses paraissent différentes et ont des dénominations différentes les unes des autres en fonction de ce qui prédomine en quantité dans le mélange des choses infinies ( $\dot{\varepsilon} \nu \tau \tilde{\eta} \mu i \xi \varepsilon l \tau \tilde{\omega} \nu \dot{\alpha} \pi \varepsilon i \rho \omega \nu)$; en effet, <disent-ils>, il n'y a pas de tout qui soit purement blanc, noir, doux, chair ou os, mais ce que chaque chose possède en plus grande quantité, c'est cela qui semble être la nature de cette chose. » (Aristote, Physique, I, 4, 187a26-b7.)

Ce raisonnement, si l'on en croit Aristote, s'appuyait sur deux prémisses : (1) rien ne naît de ce qui n'est pas (principe communément admis par les physiciens) et (2) les contraires naissent des contraires (observation empirique). Pour concilier ces deux prémisses inconciliables, la solution d'Anaxagore est de dire que, en réalité, les contraires ne naissent pas à proprement parler des contraires comme le laisse croire l'observation empirique, mais qu'ils deviennent seulement perceptibles. Autrement dit, tel contraire était déjà présent mais imperceptible, et c'est sa manifestation que nous prenons pour une « naissance ». Et comme on voit « naître » tout de tout, c'est donc que tout est dans tout (et pas seulement les contraires). Ce qui est vrai des contraires (qui constituent un cas limite) l'est a fortiori dans le cas de tout (2'). Ainsi, si tout naît de tout, c'est donc que tout est mêlé à tout. Il n'y a donc pas à proprement parler d'éléments, mais tous les matériaux qui deviennent visibles sont en fait présents en acte dans le matériau dont ils s'extraient et demeurent invisibles tant qu'ils y sont contenus $^{14}$. Ce sang n'est pas « né » de ce pain, il était présent dans ce pain mais imperceptible en raison de sa faible quantité; il est juste devenu visible. Et ce sang lui-même n'est pas simplement du sang mais un « mélange de choses infinies » dans lequel le sang prédomine, ce qui explique qu'on le nomme «sang ».

14. Il est impossible, dans les limites de cet article de rappeler toutes les hypothèses qui ont été avancées concernant les choses présentes dans le mélange (matériaux homéomères, qualités contraires, semences...) et les modalités du mélange ( $\pi \alpha \rho \dot{\alpha} \theta \varepsilon \sigma \iota \varsigma$, кр $\tilde{\alpha} \sigma \iota . .$.$) . Pour un$ état de la question, voir Curd 2007, p. 153-177. 
C'est donc bien au moyen d'un raisonnement que l'inhérence universelle est inférée. Ce raisonnement qui s'appuie sur les deux prémisses que nous avons mentionnées donne à voir ce qui est invisible (à savoir « tout est mêlé à tout »; chaque matériau est un « mélange de choses infinies » qui contient tout type de matériaux et de qualités contraires). C'est ainsi, semble-t-il, que l'on peut comprendre l'expression « parties concevables par la raison » $(\lambda \dot{o} \gamma \omega \theta \varepsilon \omega p \eta \tau \dot{\alpha} \mu \dot{o} p ı)$ que l'on trouve dans ce témoignage d'Aétius, qui présente des échos incontestables au texte d'Aristote :

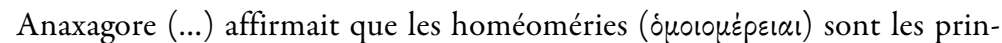
cipes des étants. (1) Il lui semblait en effet tout à fait difficile de <dire> comment quelque chose peut naître de ce qui n'est pas et être détruit en ce qui n'est pas. En tout cas, (2') nous ingérons une nourriture simple et ho-

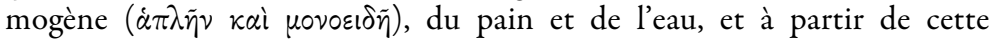
nourriture sont nourris cheveux, veines, artères, chair, nerfs et les autres parties $(\mu \dot{o} p l \alpha)$. Dans ces conditions, il faut admettre ceci : dans la nour-

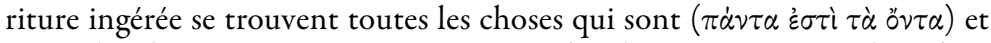
toutes les choses sont augmentées à partir des choses qui sont. Et il y a dans cette nourriture des parties ( $\mu$ ópı $\alpha$ ) capables d'engendrer du sang, des nerfs, des os, et le reste, ces parties étant concevables par la raison ( $\ddot{\alpha} \hat{\eta} \nu \lambda \partial \gamma \omega \theta \varepsilon \omega-$ $p \eta \tau \dot{\alpha} \mu \dot{o} p(\alpha)$. Car il ne faut pas tout rapporter à la sensation <en disant $>$ que ce sont le pain et l'eau qui produisent ces choses : mais <il faut dire que $>$ dans ceux-ci il y a des parties concevables par la raison $(\lambda \dot{o} \gamma \omega \theta \varepsilon \omega \rho \eta \tau \dot{\alpha}$ $\mu \dot{o}(\alpha)$. Du fait donc que les parties ( $\mu \dot{\varepsilon} p \eta)$ contenues dans la nourriture sont semblables (ö $\left.\iota_{\alpha} \alpha\right)$ aux choses engendrées, il les a appelées homéoméries et a affirmé qu'elles sont les principes des choses qui sont (...). (Aétius, Placita, I, 3, 5. La traduction suit le texte grec tel qu'il figure dans Diels-Kranz, 59A46.)

Bien que ce texte s'appuie sur un exemple (la nutrition), on y retrouve le raisonnement reconstruit par Aristote: le texte d'Aétius mentionne les deux prémisses (1) et (2') et aboutit à la même conclusion (appliquée à l'exemple) : «dans la nourriture ingérée se trouvent toutes les choses qui sont. » Mais ce qui est intéressant, c'est que ce témoignage rend explicite l'opposition entre sensation et raison. « Il ne faut pas tout rapporter à la sensation » : ce n'est pas le pain qui donne naissance au sang et à la chair, autrement dit ce n'est pas le pain qui devient chair et sang, car cela reviendrait à dire que le sang naît de ce qui n'est pas sang (le pain), hypothèse exclue par la prémisse (1). Il faut donc certes s'appuyer sur la sensation (on voit que le sang est engendré quand du pain est ingéré) mais seulement jusqu'à un certain point, c'est-à-dire autant que le permet la prémisse (1). C'est donc par le raisonnement ( $\lambda$ óyos) que l'on peut inférer 
l'existence de parties sanguines dans le pain, lesquelles sont donc « concevables par la raison $\gg$ faute d'être perceptibles par la sensation ${ }^{15}$.

Arrêtons-nous un instant sur l'étymologie qu'Aétius propose à la fin du texte. Les homéoméries d'Anaxagore sont, selon lui, « les parties ( $\mu \dot{z} p \eta)$

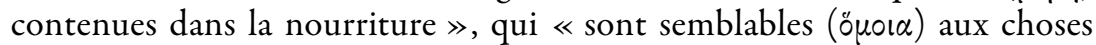
engendrées », par exemple le sang contenu dans le pain, qui est semblable au sang de l'organisme qui l'ingère. Dans cette interprétation, l'homéomère est un relatif : c'est une partie semblable à, c'est-à-dire semblable à la chose à laquelle elle va donner naissance. C'est cette similitude forte, qui est en réalité une identité, entre le sang imperceptible contenu dans le pain et le sang devenu visible dans l'organisme qui s'en est nourri, que met en évidence l'étymologie proposée par Aétius. Autrement dit, celle-ci insiste sur une identité qui se maintient à travers le temps : du sang qui coule dans ces veines était présent, bien qu'imperceptible, dans le pain. L'homéomère relie le présent au passé ; il est ce qu'il était parce qu'il était ce qu'il allait être. Il permet de remonter le temps et autorise donc le regressus sur lequel s'appuie le deuxième argument qu'Aristote attribue de façon conjecturale à Anaxagore :

Tous ceux qui posent une infinité d'éléments, comme Anaxagore et Démocrite, disent que l'infini est continu par contact, l'un le composant d'homéomères et l'autre de la réserve séminale universelle des figures. L'un (scil.

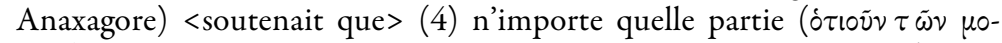

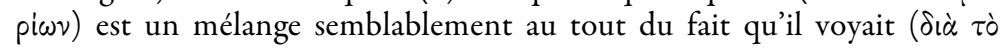
óãv) que (2') n'importe quoi naît de n'importe quoi. En effet, c'est pour

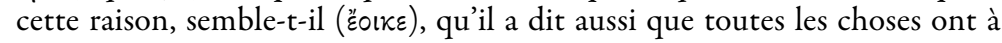
un moment été ensemble, comme cette chair-ci et cet os-ci, et ainsi n'importe quoi ; et donc toutes choses ; et par conséquent en même temps ; car il y a un commencement de la séparation non seulement dans chaque chose, mais aussi pour toutes. En effet, puisque ce qui naît naît d'un corps qui lui est semblable, et qu'il y a une génération de toutes choses, bien que pas en même temps, il faut qu'il y ait aussi un commencement de la génération, et celui-ci est unique - il l'appelle Intellect -, or c'est en s'étant mis à penser à partir d'un certain commencement que l'Intellect exerce son activité. Par conséquent, il est nécessaire que (5) toutes les choses aient été ensemble à un moment, et qu'elles aient commencé à se mouvoir à un moment. (Aristote, Physique, III, 4, 203a23-33.)

15. Pour une étude de ce texte, et plus généralement sur la question des homéomères dans la tradition doxographique, voir Lanza 1963, dont les résultats sont exposés et critiqués par Louguet 2013, qui propose une approche sensiblement différente. 
Ce texte présente deux raisonnements distincts qui s'appuient sur la même prémisse : on voit que n'importe quoi naît de n'importe quoi. C'était la prémisse (2') énoncée en Physique, I, 4, $187 \mathrm{~b} 1$ sq.

Le premier raisonnement infère de la prémisse une conclusion (4) qui n'est pas aisée à comprendre en raison de son caractère relativement abstrait et général: «n’importe quelle partie est un mélange semblablement au tout $\gg$. De quel tout et de quelle partie s'agit-il ? Le terme désignant la partie (róprov) avait deux référents distincts dans le texte d'Aétius précédemment cité : (a) le sang, les cheveux, les os, etc. qui constituent les parties de l'animal (les parties phénoménales); (b) les parties imperceptibles contenues dans le pain (les parties concevables par la raison). Cela dit, même si l'énoncé d'Aristote permet plusieurs interprétations possibles en raison de sa généralité, le contexte semble donner raison aux commentateurs anciens. Selon Simplicius et Philopon, le tout désigne l'ensemble des homéomères (dont Aristote vient de dire qu'il est infini - en grandeur - en raison du contact des homéomères infinis en nombre), et la partie désigne chaque homéomère. Aristote insisterait ainsi sur l'aspect irréductiblement composite de chaque homéomère, par opposition aux atomes démocritéens.

D’après Aristote, Anaxagore en était arrivé à cette conclusion « parce qu'il voyait que n'importe quoi naît de n'importe quoi ». Cette prémisse possède une propriété remarquable : elle peut être utilisée à la fois pour un raisonnement prospectif et pour un raisonnement rétrospectif, selon que l'on met l'accent sur le premier «n'importe quoi » ou sur le deuxième. Et il est vraisemblable que, dans le premier raisonnement, il faille entendre qu'un n'importe quoi qui semble pourtant simple donne naissance à toute sorte de choses hétérogènes (《n'importe quoi naît de n'importe quoi »); ce qui permet d'inférer que la « partie » contenait en réalité ces choses auxquelles elle a donné naissance et qu'elle est par conséquent un mélange, un tout qui contient toutes choses. Autrement dit, la partie n'est pas partielle : elle contient la totalité des espèces de matériaux; la totalité des espèces, mais sous forme d'échantillons, pourrait-on dire.

Le second raisonnement conclut, lui, au mélange universel initial de toutes choses. Dans ce mélange initial, tous les matériaux sont présents, non pas sous forme d'échantillons, mais chacun dans sa totalité. Tandis que le premier raisonnement utilisait la prémisse «n'importe quoi naît de n'importe quoi » dans un sens prospectif (la partie est elle-même un tout, qui sera à son tour un tout-origine qui donnera naissance à des parties, qui seront elles-mêmes des touts-origines pour d'autres parties, etc. : il n'y aura jamais d'élément pur), celui-ci l'utilise dans un sens rétrospectif, au moyen d'un regressus (il n'y a jamais eu d'élément pur). Puisque «n'importe quoi naît de n'importe quoi » au présent, on peut en inférer que les choses qui 
donnent naissance aux choses qu'elles contenaient étaient elles-mêmes présentes dans les choses qui leur ont donné naissance. Et puisque chaque séparation singulière est le fait de l'Intellect, Anaxagore en infère, si l'on suit la reconstruction d'Aristote, l'antériorité chronologique du mélange à chaque étape. La pensée de l'Intellect consistant à distinguer, le mélange est antérieur à son activité. Il n'y a pas d'activité séparatrice sans qu'il y ait quelque chose à séparer et donc quelque chose de mélangé. Si donc l'Intellect a commencé à penser-séparer à un moment, il est nécessaire que, avant le commencement de son activité, rien n'ait existé à l'état séparé et que, par conséquent, tout ait été mélangé.

On pourrait se demander pourquoi Anaxagore a postulé le commencement de l'activité de l'Intellect. Après tout, celui-ci aurait pu tout aussi bien exercer son activité de toute éternité. Sans doute y a-t-il à cela des raisons historiques (aucun philosophe de la nature n'a soutenu l'éternité du monde avant Aristote), mais peut-être y a-t-il une raison plus essentielle. Et c'est cette raison, semble-t-il, qu'Aristote tente d'expliciter en proposant sa reconstruction de l'argument. Chaque processus de différenciation que l'on voit à l'œuvre quotidiennement dans la nature (lorsque la plante se développe à partir d'une simple pousse ou que l'embryon prend forme à partir de la semence, etc.) est comme une micro-cosmogonie: invariablement, l'ordre est précédé de l'indistinct, l'hétérogène de l'homogène (du moins en apparence). Si n'importe quoi naît de n'importe quoi, si toute naissance est une séparation, un démêlage, une révélation de ce qui était imperceptible, alors, chaque chose donnant naissance étant elle-même née, elle-même résulte du même processus de discrimination, et ainsi de suite. Mais de même que la différenciation progresse dans chaque cas singulier (dans chaque micro-cosmogonie), de même elle doit également progresser dans le cas de la macro-cosmogonie. Et ce qui justifie cette inférence, c'est que le principe séparateur est le même dans les deux cas : l'Intellect est le principe unique (c'est-à-dire universel) de séparation. Les mêmes causes ont les mêmes effets. Ainsi, puisque la différenciation progresse au niveau macrocosmique comme elle le fait au niveau microcosmique, le mélange universel (de toutes choses en même temps) est antérieur au début de l'activité de l'Intellect. Dans l'argument tel qu'Aristote le conjecture, il y a donc ainsi, pour ainsi dire, un passage à la limite qui met un terme au regressus.

Mais, peut-on se demander de nouveau, y a-t-il une raison forte qui justifie ce passage à la limite ? Pourquoi le regressus n'est-il pas infini, puisque aussi bien le processus de séparation, lui, se poursuit indéfiniment ? Il se pourrait que la lecture de la «traduction » d'Anaxagore qu'opère Aristote en Métaphysique, A, 8 nous donne une clé. 
Si on suppose qu'Anaxagore a posé deux éléments, cette supposition sera très conforme à un raisonnement qu'il n'a certes pas articulé lui-même mais qu'il aurait néanmoins nécessairement suivi si on l'y avait amené. Même s'il est absurde de dire que toutes choses étaient mélangées à l'origine, notamment parce qu'il s'ensuit qu'il faut que ces choses aient préexisté à l'état non mélangé, et parce que par nature n'importe quoi ne se mêle pas à n'importe quoi, et en outre parce que les affections et les accidents seraient séparables des substances (car ce sont les mêmes choses qui se mélangent et qui se séparent), cependant, s'il l'on suit son raisonnement en articulant ce qu'il veut dire, il sera sans doute manifeste qu'il dit quelque chose d'assez moderne. En effet, quand rien n'était séparé, il est évident qu'on ne pouvait rien prédiquer de vrai de cette substance, je veux dire par exemple qu'elle n'était ni blanche, ni noire, ni grise, ni d'une autre couleur, mais elle était nécessairement incolore, sinon elle aurait eu une de ces couleurs; de la même façon, elle est également sans saveur, pour la même raison, et sans aucune autre propriété de ce genre ; en effet, elle n'a ni qualité ni quantité, et elle n'est pas quelque chose de déterminé; car elle aurait possédé quelqu'une des choses qu'on dit appartenir à la classe des formes, mais c'est impossible puisque toutes choses sont mélangées. En effet, cette forme aurait déjà été séparée; or il dit que toutes choses sont mêlées sauf l'Intellect, et que lui seul est sans mélange et pur. Il s'ensuit de cela qu'il dit que les principes sont l'Un (car celui-ci est simple et sans mélange) et l'Autre, tout comme nous posons l'indéterminé avant qu'il soit déterminé et qu'il participe à quelque forme, si bien que, bien qu'il ne parle ni correctement ni clairement, il veut cependant dire quelque chose qui se rapproche de ce que l'on a soutenu ensuite ou plutôt de ce que l'on croit de nos jours. (Aristote, Métaphysique, A, 8, 989a30-b21.)

Dans la «traduction » qu'il propose, Aristote voit le caractère indéterminé du matériau comme un progrès majeur accompli par Anaxagore sur ses prédécesseurs. La thèse du mélange universel est une erreur, mais une erreur fructueuse pour peu que l'on en saisisse la portée et que l'on interprète correctement l'intention d'Anaxagore. Si on suit l'esprit et non la lettre de ce qu'il a dit, il s'avère que son système se laisse appréhender comme un dualisme opposant deux principes ${ }^{16}$. Le mélange des choses infinies, dans cette lecture, n'est pas considéré comme une pluralité, mais comme une unité ${ }^{17}$. Aristote semble en effet considérer que ce mélange est un en acte et multiple en puissance, comme l'est tout mélange au sens technique du terme dans sa propre conception (voir Génération et corruption, I, 10). Aussi, dans cette lecture d'Aristote, bien que l'hypothèse du mélange initial soit erronée parce que le mélange implique un état antérieur où les

16. Cette lecture a été adoptée par Théophraste (Phys. Op. fragment 4, cité par Simplicius, in Phys. 27 et 154), qui propose de ce fait de rapprocher Anaxagore d'Anaximandre.

17. Voir aussi Métaphysique, $\Lambda, 2,1069 \mathrm{~b} 20$ sqq. 
choses étaient non pas mélangées mais séparées, elle comporte quelque chose de vrai, à savoir l'idée que l'indéterminé préexiste à ce qui relève de la forme. Il est le réceptacle de la forme, le substrat irréductible. Un substrat qui subsiste, qui ne disparaît pas une fois qu'il est informé, et qui conserve toujours toutes ses potentialités (parce qu'il n'est rien en acte, d'après la lecture d'Aristote - parce qu'il est toutes choses, d'après Anaxagore). Il est ce sans quoi l'Intellect ne pourrait pas exercer son activité (car il n'aurait rien à mettre en mouvement ni à séparer : rien sur quoi agir).

En fait, Anaxagore a bien postulé l'existence en acte d'une infinité de choses à l'origine, puisqu'il attribue leur imperceptibilité à leur petitesse (B1). Aussi, plutôt que de parler d'indétermination comme le fait Aristote, il serait plus juste de parler de surdétermination. En commençant son traité par « Toutes choses étaient ensemble, infinies en quantité et en petitesse »

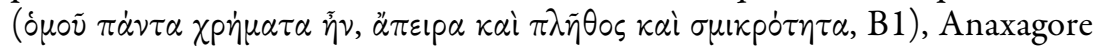
se démarque clairement de Parménide («il est maintenant, tout entier en-

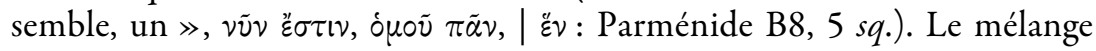
originel est une pluralité irréductible et intrinsèquement hétérogène. L'homogénéité, caractéristique de l'Étant de Parménide («il est tout entier

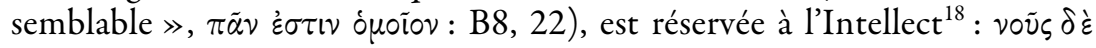

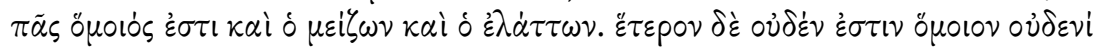
(«Et l'Intellect est tout entier semblable, aussi bien le plus grand que le plus petit. Mais rien d'autre n'est semblable à rien », B12). Cela dit, le mélange rend les différences indistinctes, comme on peut le voir dans le fragment B $4 b$ :

Mais avant cette séparation, toutes les choses étant ensemble ( $\pi \dot{\alpha} \nu \tau \omega \nu$ ó ov

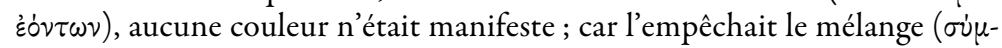
$\mu(\xi \xi))$ de toutes les choses, de l'humide et du sec, du chaud et du froid, du lumineux et de l'obscur, comme s'y trouvaient beaucoup de terre et des semences infinies en nombre qui ne se ressemblaient en rien. Car aucune des

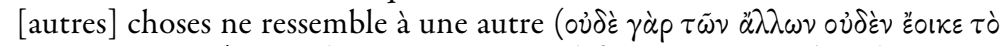
$\dot{\varepsilon} \tau \varepsilon p o v \tau \tilde{\omega} \dot{\varepsilon} \tau \dot{\varepsilon} \rho \omega)$. Les choses étant ainsi, il faut croire que dans le tout se

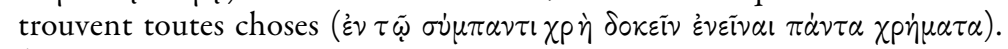
(B4b, cité par Simplicius, in Phys. 34, 21.)

Ainsi, le mélange $(\sigma \dot{u} \mu \mu \xi \xi \varsigma)$ de toutes les différences aboutit à leur imperceptibilité, ce qui justifie en un sens l'interprétation d'Aristote. La surdétermination réelle se traduit en une indétermination phénoménale. L'indétermination qu'Aristote perçoit dans le ó $\mu \delta \tilde{\nu} \pi \dot{\alpha} \nu \tau \alpha$ permet toutes les déterminations possibles. C'est un en-puissance comme l'est toute matière avant qu'elle ait reçu la forme: le mélange neutralise toutes les différences 
et n'est par conséquent que privation. L'acte de détermination n'est possible que s'il existe préalablement quelque chose à déterminer, à délimiter. De même donc qu'il y a une antériorité temporelle de la matière sur la forme, de la puissance sur l'acte, il y a également une antériorité du mélange sur l'activité de l'Intellect, parce que, selon la lecture d'Aristote, le mélange est comme une matière.

Mais pour expliquer l'antériorité du mélange sur l'activité de l'Intellect, peut-être peut-on aller encore un peu plus loin que ne le fait Aristote. Anaxagore dit en B12 : «Et l'Intellect a commandé l'ensemble de la révolution, de sorte qu'il y eût révolution au commencement. Et d'abord, la révolution a commencé par le petit, et puis la révolution augmente, et elle augmentera encore. » Autrement dit, le mouvement de révolution séparatrice initié par l'Intellect a un commencement mais pas de fin. On peut supposer que la pluralité originelle est la condition de possibilité du devenir, et l'irréductibilité de l'hétérogénéité la condition de possibilité de la perpétuation de ce devenir. La $\chi \dot{\omega} p \alpha$ du Timée, qui peut être lue en filigrane derrière «l'Autre » de Métaphysique, A, 8, pourrait faire le lien entre le mélange originel d'Anaxagore et la matière d'Aristote. Elle est dépourvue de forme, indéterminée, comme l'est la matière d'Aristote, mais elle est aussi la Nécessité à laquelle se confronte le Démiurge. L'idée que nous voudrions suggérer est que la surdétermination du mélange originel anaxagoréen présente le même type de nécessité : l'Intellect s'affronte toujours au mélange, et il ne peut faire qu'il ne soit pas. La surdétermination (l'irréductible hétérogénéité du mélange originel) fait que l'Intellect sera toujours vaincu : bien qu'il cherche à séparer, c'est-à-dire à la fois à différencier le mélange (ce qui semble homogène : l'origine), et à unifier, purifier, simplifier ce qui est mélangé (ce qui est hétérogène), il ne le peut pas (une fois pour toutes). Mais ce qui est cause de son échec est également ce qui lui permet de poursuivre indéfiniment : il ne peut pas différencier (une fois pour toutes) et le peut en même temps (indéfiniment) parce que toujours, à quelque échelle que ce soit, et à quelque moment que ce soit, toutes choses sont toujours ensemble.

Ainsi, si l'on suit cette reconstruction de l'argument que conjecture Aristote, l'homologie temporelle fonctionnerait dans les deux sens : maintenant comme à l'origine, toutes choses sont dans toute (B6), et à l'origine comme maintenant, toutes choses étaient dans toute (Physique, III, 4). Cette dernière inférence repose elle-même, comme nous avons pu le voir, sur une autre inférence énoncée en Physique, I, 4 : la génération est la manifestation de choses qui étaient là, mais imperceptibles en raison de leur petitesse. Ce principe, que nous pourrions appeler le « principe d'homéomérie » (les choses naissent de parties qui leur sont homéomères, c'est-àdire semblables à elles), est l'expression de l'homologie qualitative. 
Nous pouvons désormais conclure cet examen de l'homologie anaxagoréenne. Que l'homologie soit qualitative ou quantitative, elle traverse le temps et l'espace et est présente à tous les stades cosmiques et à toutes les échelles. Mais bien que cette loi soit universelle, elle ne se laisse pas appréhender au niveau phénoménal. Elle est donc à chaque fois inférée au moyen d'un raisonnement. Ce qui ne se voit pas et que le raisonnement révèle, c'est que les choses sont ailleurs comme ici, qu'elles sont mélangées aujourd'hui comme à l'origine et qu'elles l'étaient à l'origine comme aujourd'hui, que le petit est comme le grand et que la partie est comme le tout qu'elle va constituer. Bref, ce qui est inféré, c'est toujours la réitération du même. Or, nous allons voir que ce même qui se répète n'est autre que le mélange universel (intrinsèquement hétérogène).

\section{Pourquoi l'analogie est-elle absente ? Une hypothèse}

\subsection{Un dualisme irréductible}

Si le processus de séparation qui se poursuit indéfiniment a connu un commencement, et si Anaxagore a attribué ce processus à l'Intellect, c'est sans doute qu'il considérait toute émergence de différences comme un progrès au regard de l'informe : le visible est plus intelligible que l'invisible, le distinct l'est plus que le confus. Toute génération fait apparaître des différences que le matériau livré à lui-même n'aurait pu de lui-même engendrer, tout comme un tas de graines hétéroclites ne saurait de lui-même se soumettre à un tri. Mais une cause motrice purement mécanique (comme le vent ou le crible) serait bien insuffisante pour rendre raison de l'ordre et de la beauté du monde. Ce qu'une force mécanique est capable de séparer se cantonne en effet au quantitatif (grand-petit, lourd-léger, dense-rare); or Anaxagore parle également de formes, de couleurs et de saveurs (B4a), autrement dit de qualités. L'Intellect ne fait pas que séparer : il organise, met

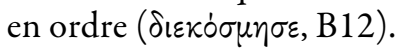

Malgré l'activité de l'Intellect, toutes choses sont toujours dans toutes. La dualité est irréductible. Mais c'est précisément cette irréductibilité qui permet la perpétuation de l'activité de l'Intellect. Le système d'Anaxagore repose sur un vrai dualisme ${ }^{19}$ : il n'y a rien de commun entre les choses et l'Intellect. Ces deux ordres sont séparés. La dichotomie qui traverse les fragments pourrait être figurée en deux colonnes à la manière de la systoichia pythagoricienne mentionnée par Aristote en Métaphysique, A, 5, $986 a 22$ sqq. :

19. Lanza 1965, p. 260-282, propose une analyse remarquable de ce dualisme. L'Intellect n'est pas l'autre des choses en tant qu'il serait incorporel, mais en tant qu'il se caractérise par des prédicats au superlatif, quand les prédicats qui qualifient les choses ne sont qu'au comparatif. L'Intellect selon Lanza représente donc un cas limite. 


$\begin{array}{ll}\text { Intellect } & \text { Choses } \\ \text { Pureté } & \text { Mélange } \\ \text { Absolu } & \text { Relatif } \\ \text { Superlatif } & \text { Comparatif } \\ \text { Identité à soi } & \text { Hétérogénéité à soi } \\ \text { Un } & \text { Infini }\end{array}$

La seule analogie que l'on pourrait trouver chez Anaxagore serait donc, à la rigueur, une analogie contrastive : l'Intellect serait aux choses ce que la pureté est au mélange, ce que l'absolu est au relatif, etc. Mais cette analogie n'est jamais énoncée explicitement; et il serait en fait plus judicieux de voir là un cas de « polarité ${ }^{20}$. Cela dit, l'intérêt réside sans doute moins dans la structure dichotomique elle-même que dans la tension dynamique engendrée par l'action de l'un sur l'autre. L'action séparatrice de l'Intellect sur les choses toujours mélangées crée un équilibre instable, une révolution qui est en même temps une évolution : une spirale qui combine le cercle (fermé) et la ligne droite (ouverte).

\subsection{L'analogie impossible}

L'analogie implique séparation et ressemblance. Pour pouvoir établir une égalité de rapports, il faut des choses distinctes qui puissent être mises en rapport : des quantités déterminées ou des qualités distinctes. Or l'inhérence universelle de toutes choses dans toutes ruine toute détermination. La matière est dépourvue de structure par elle-même. On ne peut donc même pas parler d'isomorphisme; il faudrait pour cela qu'il y ait des articulations et des choses distinctes à mettre en rapport, des consonnes, du discret. Mais chez Anaxagore, tout est voyelle, pour ainsi dire. La matière est fluide, insaisissable. L'identité est évanescente parce qu'elle ne repose sur rien de ferme ; toute unité se dissout dans l'infini qu'elle recèle. L'Intellect, qui est le même absolu, est trop autre par rapport aux autres choses pour pouvoir être mis en rapport avec elles; et les choses, qui sont l'hétérogénéité absolue, ne peuvent se rapporter qu'à elles-mêmes par un rapport homologique mais non analogique.

Un continuum quantitatif et qualitatif se laisse entrevoir dans les fragments. Le fragment B3, comme nous l'avons vu, fait état d'une continuité, et même d'une égalité entre le grand et le petit. Le fragment B8, quant à lui, fait mention d'une continuité entre qualités contraires (le chaud et le froid ne sont « pas coupés à la hache »). On pourrait objecter que la continuité entre contraires ne suffit pas à établir l'inhérence universelle de toutes choses dans toutes. Cependant, nous avons vu comment, dans le fragment B6, en s'appuyant sur l'impossibilité d'une existence 
séparée d'un plus petit, Anaxagore en venait à établir non seulement la possibilité du mélange, mais aussi sa nécessité (l'impossibilité qu’il ne soit pas). Le fragment B5 fait certes état d'une égalité des choses après leur discrimination :

Que rien n'est engendré ni détruit parmi les homéomères, mais qu'ils demeurent toujours les mêmes, il le montre en disant: « Ayant ainsi été discriminées, il faut savoir que toutes les choses ne sont en rien ni moins ni plus nombreuses (car il n'est pas réalisable qu'il y ait plus que toutes les choses), mais toutes les choses sont toujours égales. » (B5, cité par Simplicius, in Phys. 156, $9^{21}$.)

Dans sa phrase introductive, Simplicius laisse entendre qu'il s'agit des homéomères. Leur persistance à travers le processus peut être interprétée comme signifiant que le cardinal de l'ensemble qu'ils constituent est constant. Il s'agit d'une infinité spécifique dénombrable. Ces homéomères sont sans doute identifiables individuellement par la raison (sang, chair, os, or, etc.), mais dans les faits ils ne le sont pas, pour la raison dite en B6: il n'y a pas d'existence séparée. En croisant ainsi en B6 le continu quantitatif de B3 (qui est exprimé par l'égalité en $\pi \lambda \tilde{\eta} \theta 0 \varsigma$ du grand et du petit) et le discret qualitatif de B5, Anaxagore en vient en B6 à mêler inextricablement les choses hétérogènes (discrètes). L'infinie divisibilité (infini indénombrable) sert de liant au mélange des choses : elle a pour conséquence que rien n'est étanche. L'inhérence universelle est une perméabilité absolue. La divisibilité infinie, brisant toute limite, empêche toute identité, toute unité. La divisibilité s'applique partout. L'étant, qui était un, homogène et limité chez Parménide, devient infiniment multiple, hétérogène et illimité chez Anaxagore, chose que permet la divisibilité infinie du continu.

Il en résulte un prodige vertigineux, un abyme digne d'un rêve, auquel pourrait faire écho un passage du Parménide de Platon. La septième série de déductions de la deuxième partie du dialogue (164b5-165e1), qui part de l'hypothèse «si l'un n'est pas », tire ainsi les conséquences pour les autres choses :

Mais, à ce qu'il semble, chacun des amas qu'elles forment (164d) est illimité

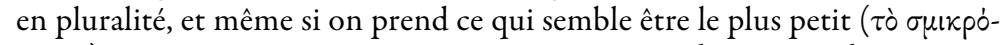
$\tau \alpha \tau \circ \nu)$, instantanément, comme si on était en train de rêver en dormant, au lieu de l'unité qu'il semblait former, cet amas apparaît être plusieurs choses, et au lieu d'être le plus petit, il apparaît extrêmement grand $(\pi \alpha \mu \mu \varepsilon \gamma \varepsilon \theta \varepsilon \varsigma)$

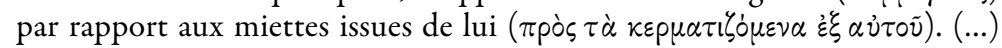

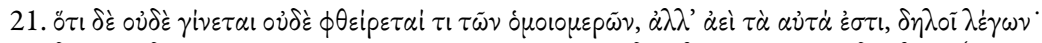

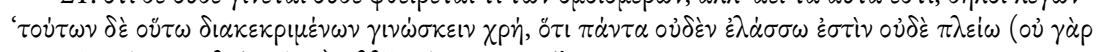

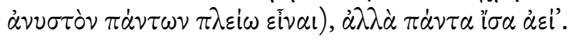


Ces choses, s'il est vrai que chacune semble être une, donneront l'impression de constituer un nombre (164e), parce qu'il y en a plusieurs. (...) En outre, certaines choses paraîtront être en nombre pair, tandis que d'autres paraîtront être en nombre impair sans que cela soit vrai dès lors qu'il n'y a pas d'un. (...) Qui plus est, il semblera, pouvons-nous dire, y avoir parmi eux un plus petit; or, ce plus petit apparaît être plusieurs choses et des choses grandes au regard de chacune des choses qui sont plusieurs, puisqu'elles sont petites (165a). (...) Et assurément chaque amas sera considéré comme égal à ces choses qui sont plusieurs et petites; en effet, un amas ne saurait changer en paraissant passer du plus grand au plus petit, avant d'avoir paru entrer dans ce qui est intermédiaire, ce qui assurément serait un simulacre d'égalité. (...) Par voie de conséquence, on l'imaginera aussi comme ayant une limite relativement à un autre amas, alors que luimême à l'égard de lui-même n'a ni commencement ni limite, ni milieu. (...) Car chaque fois qu'on appréhende par la pensée que l'une de ces déterminations appartient à l'un de ces amas, avant ce commencement apparaît toujours un autre commencement (165b), après cette fin subsiste apparemment toujours une fin différente, dans le milieu surgissent d'autres choses qui se trouvent encore plus dans le milieu que le milieu - mais plus petites - parce qu'on ne saurait appréhender ces déterminations comme appartenant à une chose en particulier, dans la mesure où l'unité n'existe pas. (...) Force est bien à mon avis que soit brisé et émietté tout ce qui est et qu'on aura appréhendé par la pensée. En effet, je suppose, c'est un amas sans unité que chaque fois on saisira. (...) Dans ces conditions, ce qui est tel ne doit-il pas paraître un à celui qui regarde de loin et qui a un regard émoussé (165c), tandis qu'à celui qui regarde de près et qui a un regard

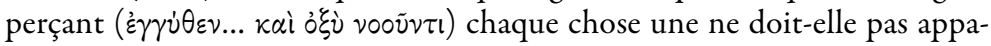
raître comme une pluralité illimitée, dès lors qu'elle est privée de l'unité, qui n'est pas? (Platon, Parménide, 164c8-165c2, trad. Brisson 1994, modifiée.)

Chez Anaxagore, l'infini est encore plus infini, pour ainsi dire, car la diversité qualitative s'ajoute au purement quantitatif. Et il semble avoir luimême dit, si l'on en croit Simplicius, que la quantité des choses qui se séparent est inconnaissable :

Mais peut-être dit-il que l'infini est insaisissable et inconnaissable pour

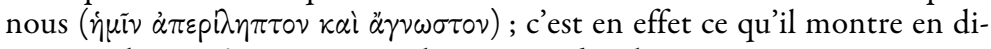
sant « si bien qu’on ne connaît la quantité des choses qui se séparent ni par la raison ni dans les faits ( $\ddot{\omega} \sigma \tau \varepsilon \tau \tilde{\omega} \nu \dot{\alpha} \pi \circ$ kp $\lambda o^{\prime} \gamma \omega \mu \dot{\eta} \tau \varepsilon$ हैp $\left.\gamma \omega\right) \gg($ (B7). En effet, qu'il croyait que les choses étaient limitées en espèces, il le montre en disant que l'Intellect les connaît toutes; et assurément, si elles étaient réellement infinies, elles seraient absolument inconnaissables; en effet, la connaissance définit et limite (ópi Łદı $\kappa \alpha$ i

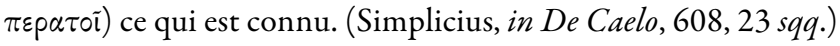


Simplicius essaie de rendre acceptable le sens de ce fragment, en confrontant ce qui y est dit avec ce qu'Anaxagore dit de l'activité de l'Intellect en B12: c'est pour nous que les choses sont inconnaissables. Nous ne

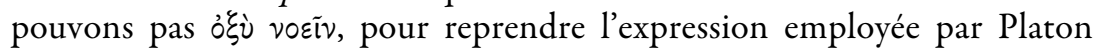
dans le texte cité ci-dessus. Mais il se peut également que les choses soient intrinsèquement inconnaissables parce que toujours mêlées. On ne peut rien saisir parce qu'il n'y a pas de limite assignable.

Comme le dit Aristote, dans un passage où il est justement question d'Anaxagore, en Physique, VIII, 1, 252a13 sq., « l'infini n'a aucun rapport

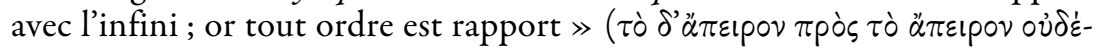

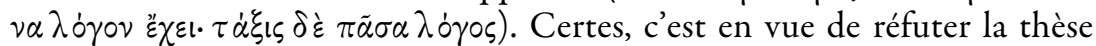
selon laquelle l'Intellect a initié le mouvement après un temps infini pendant lequel le mélange était immobile qu'Aristote émet cette objection (l'alternance entre l'immobilité et le mouvement posée par Empédocle est selon lui plus correcte car elle traduit un ordre). Néanmoins, on pourrait aussi voir dans cette phrase une description assez juste de l'état de la matière (c'est-à-dire des choses) dans le monde d'Anaxagore : alors qu'Empédocle explique la nature des choses au moyen de rapports (ce sont les différences de proportion des quatre éléments qui expliquent la diversité des choses phénoménales), on ne trouve rien de tel chez Anaxagore. Le principe de prédominance, énoncé à la fin de B12 («chaque chose est et était le plus manifestement les choses qui sont en elle les plus nombreuses »), explique l'apparence phénoménale d'une chose non pas par une combinaison d'ingrédients dont les changements de proportions conduiraient à des changements perceptibles, mais par la supériorité quantitative d'un ingrédient sur les autres. C'est en tout cas ainsi que l'a compris Aristote à la fin du texte de Physique, I, 4 que nous avons cité plus haut, et il ne manque jamais de louer la solution d'Empédocle (en vertu du principe d'économie). Autrement dit, si toutes les choses sont toujours mêlées, ce n'est pas le mélange qui détermine la nature phénoménale de telle chose particulière : le sang phénoménal semble être du sang parce qu'il contient du sang en plus grande quantité que de toutes les autres choses qu'il contient (chez Empédocle, en revanche, le sang résulte d'une combinaison particulière des quatre éléments selon certaines proportions déterminées; les mêmes éléments entrent dans la composition de l'os, mais en des proportions différentes, etc.). Aristote dit à ce propos quelque chose de très éclairant :

Même en concevant l'élément comme ils le font (scil. en posant des homéomères), il n'est pas nécessaire d'en supposer une infinité, car on aura les mêmes résultats en faisant l'hypothèse d'éléments en nombre fini. En effet, on aboutira à la même chose si on n'a que deux ou trois éléments de ce genre, comme s'efforce de le démontrer aussi Empédocle. En effet, puisque même ainsi ils n'arrivent pas à tout former à partir d'homéomères (car 
ils ne font pas un visage avec des visages, ni aucune autre des choses naturellement mises en forme <de parties identiques à elle>), il est manifeste qu'il eût été bien meilleur de rendre les principes finis en nombre, et de montrer qu'ils sont en nombre minimum avec les mêmes conséquences, comme le requièrent aussi les mathématiciens; car, eux, ils prennent toujours des principes spécifiquement ou quantitaivement définis. (Aristote, Traité du Ciel, III, 4, 302b21-30, trad. Pellegrin 2004.)

Les choses « naturellement mises en forme » ( $\tau \dot{\alpha} \kappa \alpha \tau \dot{\alpha} \phi \dot{v} \sigma \iota \dot{\varepsilon} \sigma \chi \eta \mu \alpha-$ $\tau \iota \sigma \mu \dot{\varepsilon} \nu \alpha)$ ne naissent pas comme les matériaux qui les composent. Les matériaux qui composent le visage (chair, sang, etc.) sont composés d'homéomères (au sens aristotélicien du terme), mais le visage n'est pas composé d'homéomères au sens où il n'est pas composé de parties identiques à lui ${ }^{22}$ (mais il est composé de sang, de chair, etc. qui sont, eux, des homéomères et composés d'homéomères). Pour employer la terminologie d'Aristote, les anhoméomères (visage) ne sont pas formés comme les homéomères (sang). Il y a donc chez Anaxagore une composition organisée, une structuration, une mise en forme; mais il resterait à en découvrir la cause. Le fragment B4a mentionne la naissance des hommes : ailleurs comme chez nous, des hommes se sont «solidifiés ». On pourrait dire qu'ils ont pris consistance, bien qu'ils soient composés de matériaux sans consistance et fluides. Il s'agit donc d'un processus que la matière seule ne saurait expliquer : les matériaux parfaitement mêlés ne font rien émerger d'eux-mêmes, et le mélange, hétérogène à l'extrême en raison de ses composants, mais homogénéisé par la divisibilité infinie, est amorphe. Une possibilité serait d'attribuer à l'Intellect la cause de la forme, et c'est ce que fait Aristote dans le texte de Métaphysique, A, 8 cité plus haut. Et en B12, l'Intellect ne fait pas que mettre en

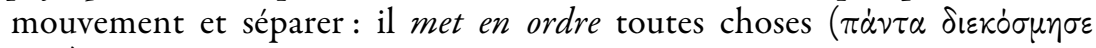
voũs).

Mais même au niveau de la génération des parties homéomères, la modalité de la séparation est assez mystérieuse. La génération est une séparation de choses qui étaient déjà là, une manifestation de choses qui étaient cachées, imperceptibles, une venue au premier $\operatorname{rang}^{23}$ de choses qui étaient latentes. Comment, dans un système qui abolit toutes limites quantitatives et qualitatives, le principe de prédominance peut-il être effectif ? Ici encore, on peut supposer que c'est l'œuvre de l'Intellect. La singularisation phénoménale est le résultat d'un changement de concentration relative. Mais dans le fond, tout est toujours mêlé, car si le sang phénoménal est composé en majorité de sang, le sang ingrédient est lui-même mêlé de

22. « Homéomère » dans ce texte semble avoir le même sens que dans le texte d'Aétius cité supra: «partie semblable à ».

23. « Magis in promptu primaque in fronte locata (Lucrèce, I, 879). 
toutes les autres choses ${ }^{24}$, comme le conjecture Philopon dans un passage de son commentaire de Physique, I, 4 :

De même que si, à partir d'un mélange de semences on extrait les grains d'orge cachés en raison de leur petit nombre et qu'on fasse un tas de grains d'orge, on n'aura pas produit une génération des grains d'orge, mais seulement leur manifestation ("ُk $\phi \alpha \nu \sigma ı \zeta)$ et leur séparation, de même pour les choses naturelles; en effet, lorsque de la chair naît l'os ou quelque chose d'autre, cela n'est pas une génération au sens propre, mais seulement une manifestation et une séparation de ce qui auparavant était caché. À ceci près que dans le cas du tas de semences mélangées il est possible de séparer une espèce pure, comme des grains d'orge, mais dans le cas des homéoméries ce n'est pas possible ; en effet, il n'est pas possible que soient jamais séparés de la chair pure, ou de la pierre ou de l'or ou autre chose, mais toujours dans tout ce qui se sépare, et quelle qu'en soit la taille, toutes choses sont présentes, mais il tire son nom de ce qui domine en lui. (Philopon, in Phys. 87, 25 sqq.)

Ainsi, en définitive, bien qu'il y ait un ordre phénoménal, une structure du monde et des choses, et que les choses soient dotées d'une identité propre, cela ne concerne que le niveau perceptible; mais au niveau infrasensible, c'est le mélange qui règne comme à l'origine. Bien qu'il y ait une évolution, grâce à l'Intellect qui rend manifeste ce qui ne l'était pas, on en revient toujours au même. L'absence d'analogie dans les textes dont nous disposons pourrait être une pure coïncidence (peut-être y avait-il des analogies dans les textes que nous avons perdus). Mais il est vraisemblable que ce ne soit pas le cas (aucun témoignage ne fait état de la moindre analogie). Tout se passe comme si Anaxagore avait voulu insister par tous les moyens sur la clôture de son système, une clôture qui s'exprime par le fait que l'inhérence universelle prend toujours le pas sur l'émergence de la singularité. Le même qui se répète est celui de la récurrence de l'hétérogénéité absolue (hétérogénéité à soi) ; et l'autre qui n'en finit pas d'émerger sous l'effet de l'Intellect est celui de l'identité à soi (-même). Si tout est dans tout, et si aucune limite quantitative ou qualitative n'est assignable, on ne peut rien mettre en rapport. Le seul véritable autre est l'Intellect ${ }^{25}$. La structure paradoxale de la matière (mise en abyme) défie le $\lambda$ ó $о \varsigma$.

La séparation ontologique résidait chez Parménide entre l'Être et les phénomènes. Anaxagore, en introduisant l'Intellect, a fait de l'Être le principe des choses sensibles : en opposant l'Intellect aux choses, il a déplacé le lieu du dualisme parménidien en même temps qu'il a rapproché dans une certaine mesure l'Être des phénomènes, en faisant de lui leur principe. 
Ainsi, l'Être infra-phénoménal (le mélange universel) se révèle être un compromis entre l'Être parménidien et les phénomènes que ce dernier ne pouvait expliquer : il est à la fois continu et homogène comme l'Être parménidien, et discret et hétérogène comme les phénomènes sensibles. Et c'est le modèle du mélange parfait, mais dans lequel les choses demeurent néanmoins en acte, qui réalise ce compromis paradoxal. Ce changement a des conséquences sur le statut de l'intellection : l'intellection qui, chez Parménide, s'identifiait à l'Être, est chez Anaxagore ce qui permet à l'Être infra-phénoménal d'accéder au niveau phénoménal, c'est-à-dire d'entrer dans le devenir en devenant principe.

En réalité (c'est-à-dire au niveau infra-phénoménal, que ce soit à l'origine ou maintenant), il n'y a ni analogie, ni proportion, ni identité. L'ordre, la beauté, la structure et les différences sont créés grâce au tri et à la composition effectués par l'Intellect: la composition harmonieuse, l'assemblage ordonné des matériaux créant le macrocosme et le microcosme nécessitent au préalable une séparation, un tri de ces matériaux mêlés dans le mélange indifférencié. La synthèse implique une analyse préalable. L'action de l'Intellect pourrait ainsi se présenter comme consistant à faire passer les choses d'un mélange à un autre mélange : d'un état de mélange indifférencié (inhérence universelle infra-phénoménale) à une combinaison ${ }^{26}$ organique structurée (identité phénoménale). Mais, alors que les formes, les couleurs, les saveurs, les grandeurs se laissent percevoir et sans doute comparer (et mettre en rapport) dans le monde des phénomènes, rien de tel en réalité au niveau infra-phénoménal où règne invariablement l'inhérence universelle, qui est à la fois obstacle et condition de possibilité de l'émergence de l'ordre via l'Intellect.

L'analogie et l'image font voir. Et ce qui est visible est l'œuvre de l'Intellect. Mais l'infra-phénoménal est aussi infra-rationnel; le mélange universel sous-jacent est fondamentalement irrationnel et confus, mais il est nécessairement. L'hypothèse que nous voudrions proposer est que les différentes occurrences de l'homologie sont destinées à insister sur le caractère paradoxal de la matière. L'analogie en revanche aurait pour effet d'introduire de l'ordre dans ce qui en est dépourvu. Comme le montre B12, l'Intellect a beau séparer et mettre en ordre, les choses sont et seront toujours mélangées. C'est dans le mélange que se trouve la permanence. C'est lui qui demeure et demeurera nécessairement quoi qu'il arrive. D'où l'insistance avec laquelle Anaxagore mentionne le principe d'inhérence universelle (pas moins de huit occurrences dans les fragments qui nous sont parvenus, ce qui est énorme).

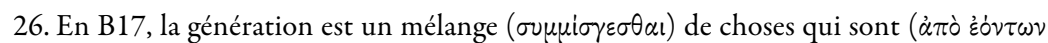

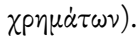


Si la répétition du même (mélange irréductible) dans les fragments est une façon de traduire en mots l'irréductibilité de l'irrationnel, qui est en même temps la condition de possibilité de la perpétuation de l'activité de l'Intellect, on peut dire qu'Anaxagore a bien calculé et subtilement œuvré : l'interprète, finalement, est face à ses fragments comme l'Intellect face à la confusion du mélange. Confronté à un irrationnel irréductible, il ne peut s'empêcher pourtant d'essayer de le dominer, en vain car toujours la confusion reprend le dessus. Et, bien que l'interprétation progresse, il a le sentiment que la réalité demeurera toujours aussi complexe. 


\section{BIBLIOGRAPHIE}

Brennan, T. 1995 : « The Text of Anaxagoras Fragment DK 59 B22 », American Journal of Philology, 116 (1995), p. 533-537.

CURD, P. 2007 : Anaxagoras of Clazomenae. Fragments and Testimonia. A Text and Translation with Notes and Essays, Toronto, 2007 (Phoenix Supplementary volume, 44 $=$ The Phoenix Presocratics. 6).

Diels, H. \& W. KRANZ 1951 : Die Fragmente der Vorsokratiker, Berlin, $1951^{6}$.

LANZA, D. 1963 : « Le omeomerie nella tradizione dossografica anassagorea », La Parola del Passato, 18 (1963), p. 256-293.

- 1965 : «Il pensiero di Anassagora », Memorie dell'Istituto Lombardo, Cl. di Lettere, 29 (1965), p. 223-288.

- 1966 : Anassagora, Testimonianze e frammenti, Florence, 1966 (Biblioteca di Studi Superiori, 52).

LLOYD, G.E.R. 1966 : Polarity and Analogy, Two Types of Argumentation in Early Greek Thought, Cambridge, 1966.

LOUGUET, C. 2002 : « Note sur le fragment B4a d'Anaxagore : pourquoi les autres mondes doivent-ils être semblables au nôtre ? », dans A. Laks \& C. Louguet (éd.), Qu'est-ce que la philosophie présocratique ?, Villeneuve d'Ascq, 2002, p. 497-530 (Cahiers de philologie, 20. Série Apparat critique).

- 2013: «L'Anaxagore de Diego Lanza: quelques réflexions», dans Ph. Rousseau \& R. Saetta Cottone (éd.), Diego Lanza, lecteur des auvres de l'Antiquité. Poésie, philosophie, histoire de la philologie, Villeneuve d'Ascq, 2013, p. 51-84 (Cahiers de philologie, 29. Série Apparat critique).

PEPE, L. 2002: «Le livre d'Anaxagore lu par Platon », dans M. Dixsaut \& A. Brancacci (éd.), Platon source des présocratiques: exploration, Paris, 2002, p. 107-128 (Bibliothèque d'histoire de la philosophie).

SCHOFIELD, M. 1980 : An Essay on Anaxagoras, Cambridge, 1980.

SEDLEY, D. 2007 : Creationism and Its Critics in Antiquity, Berkeley/Los Angeles/Londres, 2007 [chap. I « Anaxagoras », p. 1-30] (Sather Classical Lectures, 66).

SIDER, D. 2005 : The Fragments of Anaxagoras: Edited with an Introduction and Commentary, $2^{\mathrm{c}}$ éd. rév. Sankt Augustin, 2005 (International pre-Platonic studies, 4). [1 $1^{\text {re }}$ éd. Meisenheim, 1981 (Beiträge zur Klassische Philologie, 118).] 